\title{
PFGE, Lior serotype, and antimicrobial resistance patterns among Campylobacter jejuni isolated from travelers and US military personnel with acute diarrhea in Thailand, 1998-2003
}

Oralak Serichantalergs ${ }^{1,2^{*}}$, Piyarat Pootong ${ }^{1}$, Anders Dalsgaard ${ }^{2}$, Ladaporn Bodhidatta ${ }^{1}$, Patricia Guerry ${ }^{3}$, David R Tribble ${ }^{4}$, Sinn Anuras ${ }^{5}$, Carl J Mason ${ }^{1}$

\begin{abstract}
Background: Campylobacter jejuni is a major cause of gastroenteritis worldwide. In Thailand, several strains of C. jejuni have been isolated and identified as major diarrheal pathogens among adult travelers. To study the epidemiology of C. jejuni in adult travelers and U.S. military personnel with acute diarrhea in Thailand from 1998-2003, strains of C. jejuni were isolated and phenotypically identified, serotyped, tested for antimicrobial susceptibility, and characterized using pulsed-field gel electrophoresis (PFGE).
\end{abstract}

Results: A total of 312 C. jejuni isolates were obtained from travelers $(n=46)$ and U.S. military personnel $(n=266)$ in Thailand who were experiencing acute diarrhea. Nalidixic acid and ciprofloxacin resistance was observed in 94.9\% and $93.0 \%$ of the isolates, respectively. From 2001-2003, resistance to tetracycline (81.9\%), trimethoprimsulfamethoxazole (57.9\%), ampicillin (28.9\%), kanamycin (5.9\%), sulfisoxazole (3.9\%), neomycin (2.0\%), and streptomycin (0.7\%) was observed. Combined PFGE analysis showed considerable genetic diversity among the C. jejuni isolates; however, four PFGE clusters included isolates from the major Lior serotypes (HL: 36, HL: 11, HL: 5, and HL: 28). The PFGE analysis linked individual C. jejuni clones that were obtained at U.S. military exercises with specific antimicrobial resistance patterns.

Conclusions: In summary, most human C. jejuni isolates from Thailand were multi-resistant to quinolones and tetracycline. PFGE detected spatial and temporal C. jejuni clonality responsible for the common sources of Campylobacter gastroenteritis.

\section{Background}

Campylobacter jejuni is a major cause of gastroenteritis worldwide, especially in children, travelers, and military personnel deployed to developing countries [1-4]. In recent years, a high prevalence of infection and an increased resistance to the antimicrobials used to treat diarrhea have been documented [5,6]. C. jejuni and C. coli can be phenotypically characterized by growth characteristics, biochemical reactions, and hippurate hydrolysis [7]. Serotyping techniques for $C$. jejuni and C. coli have been developed [8-10]. Molecular

\footnotetext{
* Correspondence: oralaks@afrims.org

'Department of Enteric Diseases, Armed Forces Research Institute of Medical

Sciences, 315/6 Rajvithi Road, Phayathai, Bangkok 10400, Thailand

Full list of author information is available at the end of the article
}

techniques such as RFLP, RAPD, PFGE, AFLP, and MLST have also been applied to $C$. jejuni isolate characterization [11].

PFGE is a well-known technique standardized by the Centers for Disease Control and Prevention (CDC) for subtyping Salmonella spp., Shigella spp., and Vibrio spp., in addition to C. jejuni $[12,13]$. Unlike other enteric bacteria, Campylobacter is a genetically diverse organism that undergoes intra- and inter-genomic exchange. However, PFGE is considered to be the most discriminatory method of characterizing C. jejuni and C. coli and identifying specific Campylobacter spp. in outbreak studies [14-16]. Furthermore, the combination of PFGE and other typing techniques can identify 
common sources of Campylobacter and other bacterial infections [17-19].

In Thailand, C. jejuni was isolated and identified as a major diarrheal pathogen among children and adult travelers, including U.S. military personnel [20-23]. A high prevalence of infection with fluoroquinolone-resistant C. jejuni was previously reported in both Thai children and U.S. military personnel $[3,24]$. However, epidemiological data on Campylobacter infection among travelers and expatriates as well as their susceptibility to other antimicrobials have not been described in Thailand in several years. This prompted us to investigate and characterize the $C$. jejuni isolates responsible for gastroenteritis in adult travelers by combining antimicrobial resistance data, serotype classification, and PFGE.

\section{Results and Discussion}

Frequency and distribution of $C$. jejuni serotypes

From a total of 312 C. jejuni isolates obtained in this study, 266 isolates were from U.S. soldiers and 46 were from foreign travelers seen at Bumrungrad Hospital, Bangkok. The prevalence of C. jejuni from diarrheal cases was detected as $11.0 \%(46 / 417)$ in the Bumrungrad Hospital study and 30.4\% (266/875) in samples from U.S. military personnel in the Cobra Gold exercises of 1998-2003 (Table 1). Overall, a total of 16 Lior serotypes were detected; the three most common serotypes were HL: 36, HL: 11, and HL: 5; these serotypes accounted for $25.0 \%$ (78/312), 13.8\% (43/312), and 7.4\% (23/312) of all of the isolates, respectively. Untypable strains composed $17.6 \%$ (55/312) of the isolates. The distribution of the serotypes and the incidence of $C$. jejuni isolates among foreign travelers and U.S. military personnel are shown in Table 2 . The two most common serotypes by study location and year are shown in Table 1.

The incidence of the serotypes described herein were included from the first to tenth ranks among the global isolates [25] but were slightly different from those reported previously in Thai children [24]. For example, serotype HL: 5 was detected infrequently among Thai children [24]. In this study, serotype HL: 5 was more common, and serotypes HL: 9 and HL: 2 were less common. The majority of $C$. jejuni isolates from serotype HL: 5 (11/23) were isolated in 2001, and most $C$. jejuni HL: 19 isolates (15/17) were obtained in 1999. The untypable isolates comprised $54.3 \%(25 / 46)$ of the isolates from foreign travelers in 2001-2002 compared to $11.3 \%(30 / 266)$ of the isolates from U.S. military personnel in this study. The adult travelers from Bumrungrad Hospital spanned several nationalities, including Japanese, European, American, and Australian. The high percentage of untypable isolates might reflect the diverse foods consumed by these travelers, while the low percentage of untypable isolates in the military personnel might reflect the limited diet consumed by U.S. military personnel on deployment.

\section{PFGE analysis of $C$. jejuni isolates}

At an $80 \%$ similarity level, a dendrogram combining data for all 312 C. jejuni isolates from Thailand was clustered into 62 genotypes (Figure 1). Four major genotypes composed $49.7 \%$ (155/312) of the C. jejuni isolates in this study, and 30 of the 62 genotypes included only a single $C$. jejuni isolate. Of the 55 isolates that were untypable by Lior serotyping, 42 could be grouped into a genotype with other known serotypes.

\section{Antimicrobial susceptibility tests}

In 1998-2003, 94.9\% (296/312) and 93.0\% (289/311) of the isolates were resistant to quinolones (NAL and CIP), but $99.0 \%(306 / 309)$ were susceptible to macrolides (ERY and AZM). The high prevalence of quinolone (NAL and CIP) resistance and macrolide (ERY and AZM) sensitivity is consistent with previously reported results from Thailand $[21,24,26]$. For the 138 C. jejuni isolates from 2001-2003, the percentages of resistant isolates detected were as follows (Table 3): CF, 100\% (138/ 138); TE, 81.9\% (113/138); SXT, 58.0\% (80/138); AMP, $30.4 \%$ (42/138); KM, 6.5\% (9/138); SU, 3.6\% (5/138); $\mathrm{NM}, 2.2 \%(3 / 138)$; and SM, $0.7 \%(1 / 138)$. None of these 138 isolates were resistant to GM, CM or CL. Notably, tetracycline resistance was also detected in over $80 \%$ of

Table 1 Numbers of $C$. jejuni isolates and two most common Lior serotypes by study location/year

\begin{tabular}{|c|c|c|c|c|}
\hline Year & Study location & $\begin{array}{l}\text { Enrolled diarrhea cases } \\
\quad(\text { Total }=1292)\end{array}$ & $\begin{array}{c}\text { Number of } C \text {. jejuni isolates } \\
\quad(\text { Total }=312)\end{array}$ & $\begin{array}{l}\text { Two most common Lior serotypes } \\
\text { (Number of isolates) }\end{array}$ \\
\hline \multirow[t]{2}{*}{1998} & Kanjanaburi & 166 & 10 & $\mathrm{HL}: 11$ (3), HL: 36 (2) \\
\hline & Cholburi & & 12 & HL: 36 (3), HL: 11 (2) \\
\hline 1999 & Korat & 198 & 84 & HL: 36 (33), HL: 19 (15) \\
\hline 2000 & Nakornsrithammarat & 256 & 68 & HL: 11 (23), HL: 36 (19) \\
\hline 2001 & Phitsanulok & 153 & 54 & HL: 5 (11), HL: 36 (8) \\
\hline $2001-2002$ & Bangkok & 417 & 46 & untypable (35), HL:11 (5) \\
\hline 2002 & Sakaew & 54 & 15 & HL: 36 (7), untypable (4) \\
\hline 2003 & Pranburi & 48 & 23 & HL: 4 (7), HL: 36 (3) \\
\hline
\end{tabular}


Table 2 Lior serotype distribution of C. jejuni isolates among foreign travelers and U.S. military personnel (1998-2003)

\begin{tabular}{|c|c|c|c|}
\hline Lior serotype & $\begin{array}{l}\text { Serotypes in foreign travelers \% }(n) \\
(\text { Total }=46)\end{array}$ & $\begin{array}{l}\text { Serotypes in U.S. military personnel \% (n) } \\
\text { (Total = 266) }\end{array}$ & $\begin{array}{l}\text { \% Total }(n) \\
(\text { Total }=312)\end{array}$ \\
\hline $\mathrm{HL}: 36$ & $6.5(3)$ & $28.2(75)$ & $25.0(78)$ \\
\hline HL: untypable & $54.3(25)$ & $11.7(30)$ & $17.6(55)$ \\
\hline HL: 11 & $6.5(3)$ & $14.7(40)$ & $13.8(43)$ \\
\hline HL: 5 & $4.3(2)$ & $7.9(21)$ & $7.4(23)$ \\
\hline HL: 1 & 0.0 & $7.5(19)$ & $6.1(19)$ \\
\hline $\mathrm{HL}: 4$ & $4.3(2)$ & $5.6(15)$ & $5.4(17)$ \\
\hline HL: 19 & 0.0 & $6.0(17)$ & $5.4(17)$ \\
\hline $\mathrm{HL}: 7$ & $2.2(1)$ & $4.9(13)$ & $4.5(14)$ \\
\hline HL: 114 & $4.3(2)$ & $4.1(10)$ & $3.8(12)$ \\
\hline HL: 28 & $4.3(2)$ & $3.8(10)$ & $3.8(12)$ \\
\hline HL: 102 & $2.2(1)$ & $1.9(5)$ & $1.9(6)$ \\
\hline $\mathrm{HL}: 2$ & 0.0 & $1.9(5)$ & $1.6(5)$ \\
\hline HL: 9 & 0.0 & $1.5(4)$ & $1.3(4)$ \\
\hline HL: 17 & $4.3(2)$ & $0.4(1)$ & $1.0(3)$ \\
\hline $\mathrm{HL}: 41$ & $2.2(1)$ & 0.0 & $0.3(1)$ \\
\hline HL: 53 & 0.0 & $0.4(1)$ & $0.3(1)$ \\
\hline HL: 6 & $2.2(1)$ & 0.0 & $0.3(1)$ \\
\hline HL: 86 & $2.2(1)$ & 0.0 & $0.3(1)$ \\
\hline
\end{tabular}

isolates that were similar to $C$. jejuni isolates from Taiwan (95\%) [27], Korea (87\%) [6,28], Canada, and the U.S. (50\%) [29,30].

The two most common resistance patterns observed in these 138 isolates were multiple resistance to four antimicrobials (NAL, CIP, CF, and TE), observed in $79.0 \%(109 / 138)$ of the isolates, and multiple resistance to five antimicrobials (NAL, CIP, CF, TE, and SXT), detected in $47.8 \%(66 / 138)$ of the isolates. Another resistance pattern (NAL, CIP, CF, TE, SXT, and AMP) was found in $15.9 \%(22 / 138)$ of the isolates, and a fourth pattern of resistance (NAL, CIP, CF, TE, and KM) was detected in $5.8 \%(8 / 138)$ of the isolates. The first common pattern was similar to a previous report of antimicrobial resistance to NAL, CIP, and TE in 53\% of clinical C. jejuni isolates in Thailand [31]. These results confirm widespread quinolone and tetracycline resistance among $C$. jejuni isolates from traveler's diarrhea in Thailand.

Antimicrobial resistance by study location and the results of the chi-square test are shown in Table 3. Interestingly, KM-resistant isolates were detected in $6.5 \%(9 / 138)$ of the isolates, but there was a significant difference (from $2.2 \%$ to $21.8 \%$ ) in the frequency of resistance in isolates from different locations $(\mathrm{p}<$ 0.001). The percentage of AMP-resistant isolates also varied by location $(\mathrm{p}<0.05)$. The resistance of isolates to SXT varied greatly from $90.7 \%$ at Phitsanulok to $58.7 \%$ at Bumrungrad Hospital, 26.7\% at Sakaew, and to undetectable levels in isolates from Pranburi $(\mathrm{p}<0.001)$. The finding of differences in AMP, KM, and SXT resistance among C. jejuni isolates from selected sites should not be considered as indicative of significant changes over time in Thailand.

In this study, the antimicrobial susceptibility tests were performed by disk diffusion assay. Other methods, including agar dilution and broth micro dilution methods, and epsilometer test (E-tests) have been used by different laboratories to measure antimicrobial susceptibilities for Campylobacter spp. [32-35]. However, good agreement of antimicrobial susceptibility test between disk diffusion and agar dilution tests has been observed in several classes of antimicrobials especially quinolone/ fluoroquinolones and aminoglycosides suggesting that disk diffusion test could be used as qualitative assay but not quantitative assay for antimicrobial susceptibility among Campylobacter spp. [36]. Although another study suggested that interpretation of erythromycin by disk diffusion assay was unreliable and should be confirmed by MIC-based methods [37], but our previous data showed high correlation of antimicrobial susceptibility by disk diffusion and agar dilution tests in erythromycin and azithromycin-resistant $C$. jejuni and C. coli isolates [38].

\section{PFGE and antimicrobial resistance by study location}

Dendrograms A thru D in Figs. 2, 3, 4, and 5 were generated for four study locations from 2001-2003. Dendrogram A includes 46 isolates obtained from Bumrungrad Hospital, Bangkok, over the two-year period from 20012002 (Figure 2). Although the common resistance pattern (NAL, CIP, CF, and TE) was observed in $76.1 \%$ 


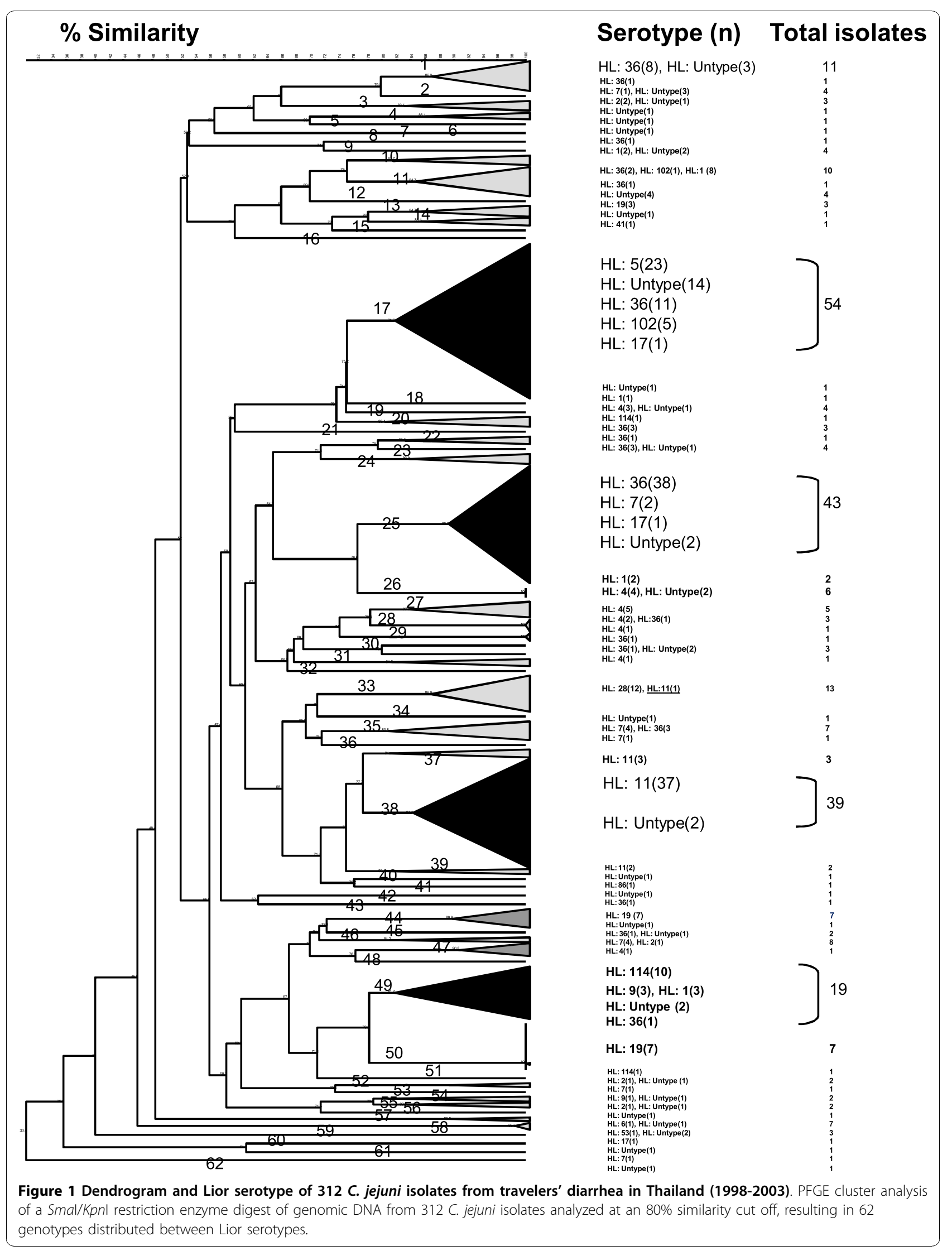


Table 3 Antimicrobial resistance among 138 human C. jejuni isolates from Thailand (2001-2003)

\begin{tabular}{|c|c|c|c|c|c|}
\hline \multirow[t]{2}{*}{ Antimicrobial } & \multicolumn{5}{|c|}{ Percentage of resistant isolates (number of isolates) } \\
\hline & $\begin{array}{l}\text { Phitsanulok } 2001 \\
(n=54)\end{array}$ & $\begin{array}{l}\text { Bangkok 2001-2002 } \\
(n=46)\end{array}$ & $\begin{array}{l}\text { Sakaew } 2002 \\
(n=15)\end{array}$ & $\begin{array}{l}\text { Pranburi } 2003 \\
(n=23)\end{array}$ & p-value * \\
\hline Nalidixic acid & $96.2 \%(52)$ & $93.5 \%(43)$ & $86.6 \%(13)$ & $100.0 \%(23)$ & NS \\
\hline Ciprofloxacin & $96.2 \%(52)$ & $87.0 \%(40)$ & $86.6 \%(13)$ & $91.3 \%(21)$ & NS \\
\hline Erythromycin & $0.0 \%(0)$ & $6.5 \%(3)$ & $0.0 \%(0)$ & $0.0 \%(0)$ & NS \\
\hline Azithromycin & $0.0 \%(0)$ & $6.5 \%(3)$ & $0.0 \%(0)$ & $0.0 \%(0)$ & NS \\
\hline Tetracycline & $90.7 \%(49)$ & $76.0 \%(35)$ & $86.6 \%(13)$ & $69.5 \%(16)$ & NS \\
\hline $\begin{array}{l}\text { Trimethoprim- } \\
\text { sulfamethoxazole }\end{array}$ & $90.7 \%(49)$ & $58.7 \%(27)$ & $26.7 \%(4)$ & $0.0 \%(0)$ & $<0.001$ \\
\hline Ampicillin & $31.5 \%(17)$ & $30.4 \%(14)$ & $60.0 \%(9)$ & $8.7 \%(2)$ & $<0.05$ \\
\hline Kanamycin & $0.0 \%(0)$ & $2.2 \%(1)$ & $20.0 \%(3)$ & $21.8 \%(5)$ & $<0.001$ \\
\hline Sulfisoxazole & $0.0 \%(0)$ & $10.9 \%(5)$ & $0.0 \%(0)$ & $0.0 \%(0)$ & NA \\
\hline Neomycin & $0.0 \%(0)$ & $2.2 \%(1)$ & $1.7 \%(1)$ & $1.7 \%(1)$ & NA \\
\hline Streptomycin & $0.0 \%(0)$ & $0.0 \%(0)$ & $1.7 \%(1)$ & $0.0 \%(0)$ & NA \\
\hline Gentamicin & $0.0 \%(0)$ & $0.0 \%(0)$ & $0.0 \%(0)$ & $0.0 \%(0)$ & NA \\
\hline Colistin & $0.0 \%(0)$ & $0.0 \%(0)$ & $0.0 \%(0)$ & $0.0 \%(0)$ & NA \\
\hline Chloramphenicol & $0.0 \%(0)$ & $0.0 \%(0)$ & $0.0 \%(0)$ & $0.0 \%(0)$ & NA \\
\hline Cephalothin & $100.0 \%(52)$ & $100.0 \%(60)$ & $100.0 \%(15)$ & $100.0 \%(23)$ & NA \\
\hline
\end{tabular}

* Pearson chi-square Monte Carlo (two-tailed); NS, non-significant; NA, not applicable.

(35/46) of the isolates, there was substantial heterogeneity in the PFGE patterns (Figure 2). The PFGE analysis suggests diverse genotypes. No specific multi-resistant antimicrobial patterns were noted in the remainder of these isolates. The dendrogram and the diversity of serotypes and antimicrobial susceptibility patterns suggest that these isolates are heterogeneous and clonally diverse.

Dendrogram B includes the 54 isolates collected from U.S. soldiers in Phitsanulok in 2001 (Figure 3). As above, $87 \%$ (47/54) of these isolates were generally multi-resistant to NAL, CIP, CF, and TE; in addition, $87 \%$ (47/54) were resistant to NAL, CIP, CF, and SXT. The genotype B3 included 24 isolates exhibiting $86.1 \%$ similarity that belonged to three serotypes: HL: 5 (11), HL: 102 (3), and HL: untypable (10). A subset of 12 isolates (B3a) within the genotype B3 had 99.7\% similarity and resistance to AMP, suggesting clonality (Figure 3). Similarly, genotype B1 consisted of five isolates of serotype HL: 36 with $100 \%$ similarity and an identical antimicrobial resistance pattern (NAL, CIP, CF, TE, and $\mathrm{SXT}) . C$. jejuni isolates in genotypes $\mathrm{B} 1$ and $\mathrm{B} 3 \mathrm{a}$ were isolated over a 5-day period and 8-day period, respectively.

Dendrogram C (Figure 4) shows 15 isolates collected from U.S. soldiers in Sakaew over a 1-month period in 2002. Genotype C1 includes 5 isolates of serotype HL: 36 with $99.2 \%$ similarity and an identical antimicrobial resistance pattern (NAL, CIP, CF, AMP, and TE), which also suggests clonality. The other 10 isolates had $<67.5 \%$ similarity and included five serotypes and seven antimicrobial resistance patterns, suggesting more diverse sources (Figure 4). All C. jejuni isolates in genotype C1 were isolated over a period of 6 days.

Dendrogram D (Figure 5) shows the 23 isolates collected from U.S. soldiers in Pranburi during a 1-month exercise in 2003 and genotype D1 includes a unique cluster of five serotype HL: 4 isolates with an unusual multi-resistant antimicrobial pattern (NAL, CIP, CF, $\mathrm{KM}$, and TE), suggesting clonality for these isolates. Similar to dendrograms B and C, genotype D1 included C. jejuni isolates collected during a 10-day period.

\section{Conclusions}

In summary, our study demonstrates the usefulness of PFGE in local epidemiological studies or in the study of small outbreaks occurring over a short time interval rather than in the long-term epidemiological studies that have been studied by others [26]. This study confirmed the existence of common $C$. jejuni clones that are associated with specific serotypes and multiple antimicrobial resistance patterns in the Cobra Gold military exercises but not in the traveler's diarrhea study at Bumrungrad Hospital. A possible explanation for these findings is that the Cobra Gold military exercises took place at particular locations with short durations (1 month). Diarrhea cases among soldiers might also be expected to be caused by common exposures. Our finding of an association between PFGE and serotype with the antimicrobial resistance patterns in these exercises differed from other studies in which the correlation between PFGE and multi-antimicrobial resistance was low $[28,39,40]$. In the Bumrungrad Hospital study, where diarrhea cases occurred in diverse populations 


\section{Dendrogram A}

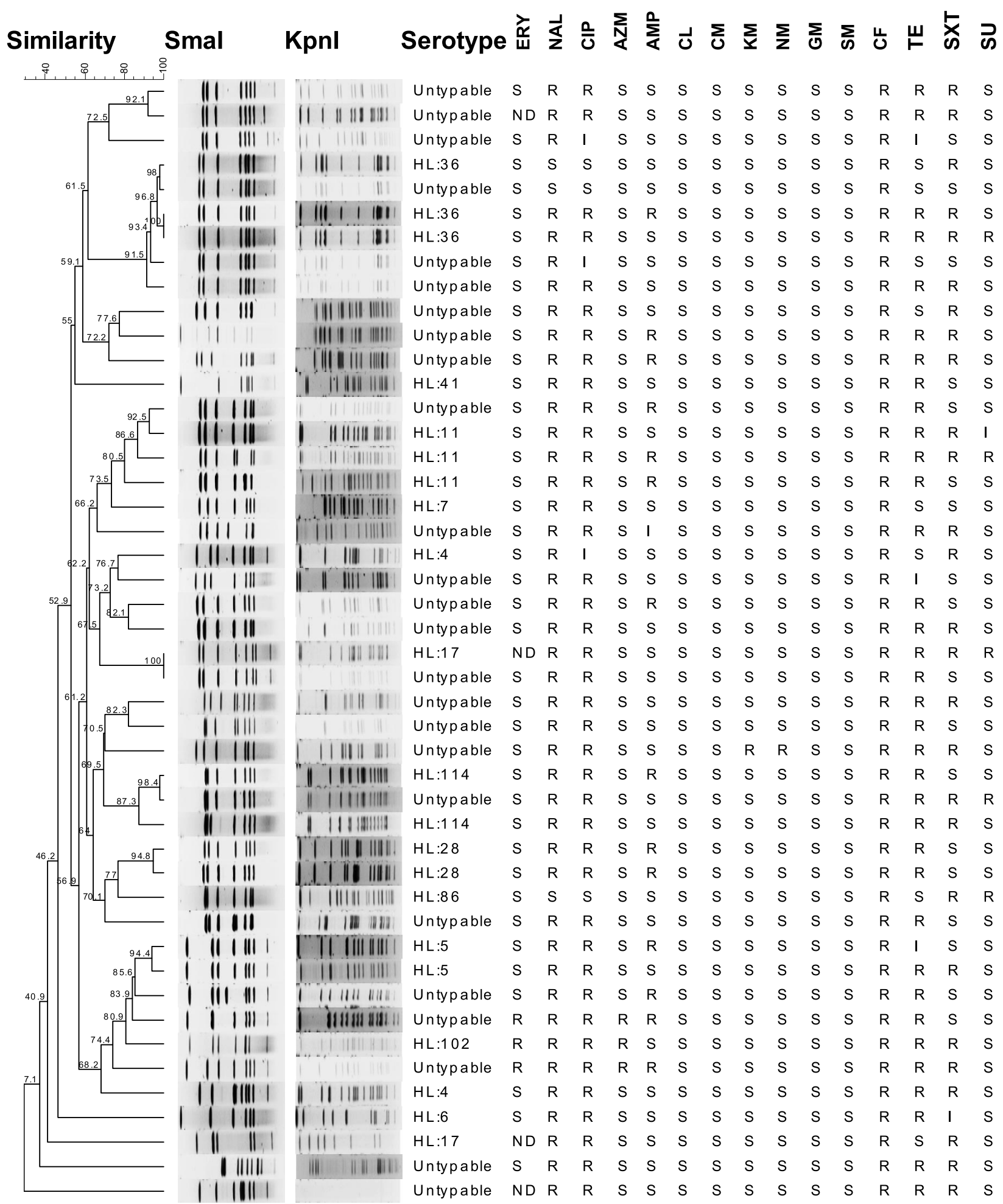

Figure 2 Dendrogram of C. jejuni isolates with Lior serotypes and antimicrobial resistance from Bumrungrad Hospital (2001-2002) Dendrogram A: PFGE cluster analysis of a Smal/Kpnl restriction enzyme digest of genomic DNA from 46 C. jejuni isolates with marked clonal heterogeneity, unrelated antimicrobial resistance patterns, and 25 untypable isolates from this study site. R, resistance; I, intermediate susceptibility; S, susceptible; ND, not tested. 


\section{Dendrogram B}

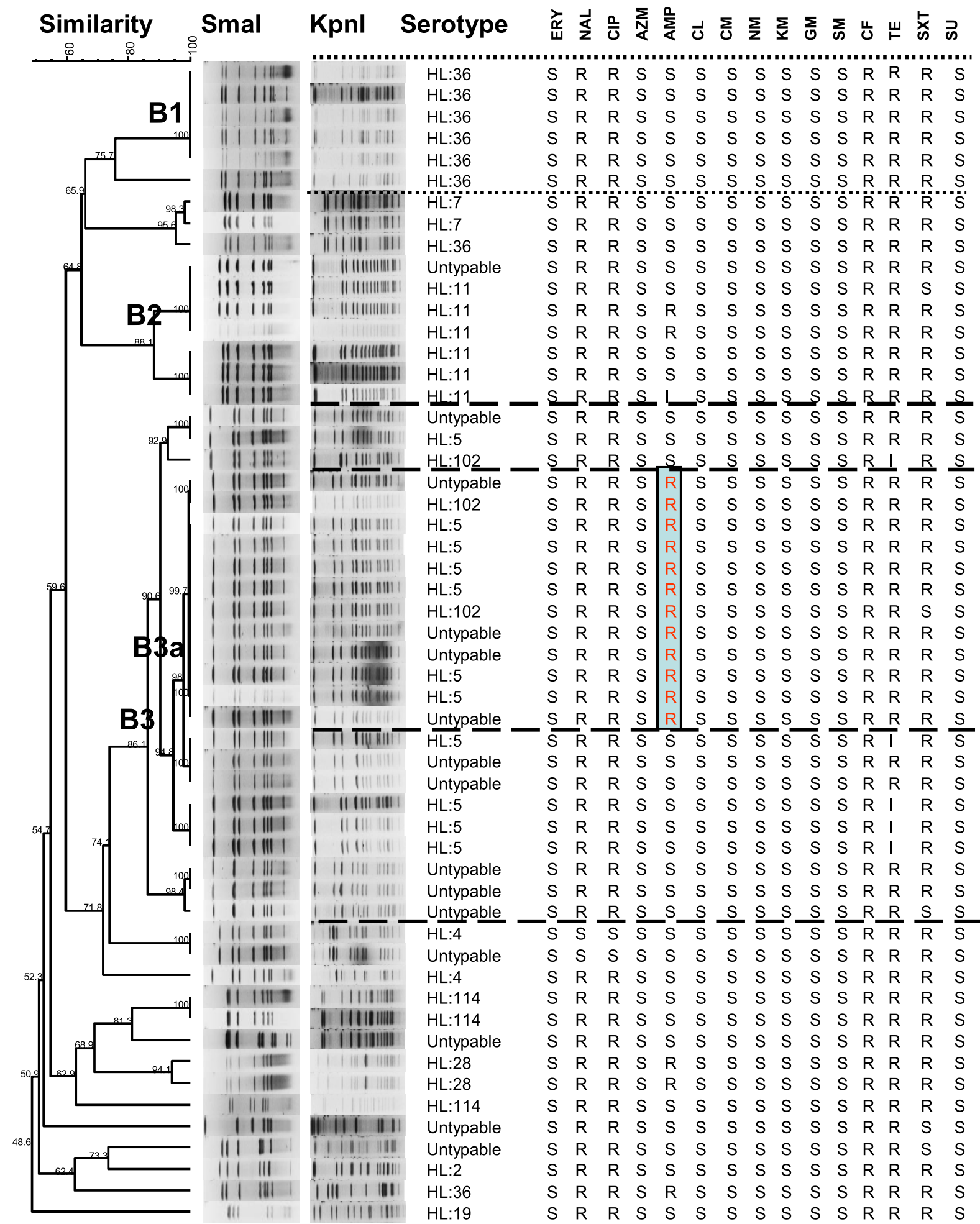

Figure 3 Dendrogram of 54 C. jejuni isolates with Lior serotypes and antimicrobial resistance from Phitsanulok 2001. Dendrogram B: PFGE cluster analysis of a Smal/Kpnl restriction enzyme digest of genomic DNA from 54 C. jejuni isolates from the Cobra Gold exercises (Phitsanulok, 2001) with evidence of clonality due to similarity in the PFGE patterns, Lior serotype(s) and related specific antimicrobial susceptibility patterns (genotypes B1 and B3a). R, resistance; I, intermediate susceptibility; S, susceptible. 


\section{Dendrogram C}

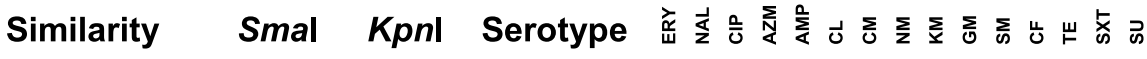

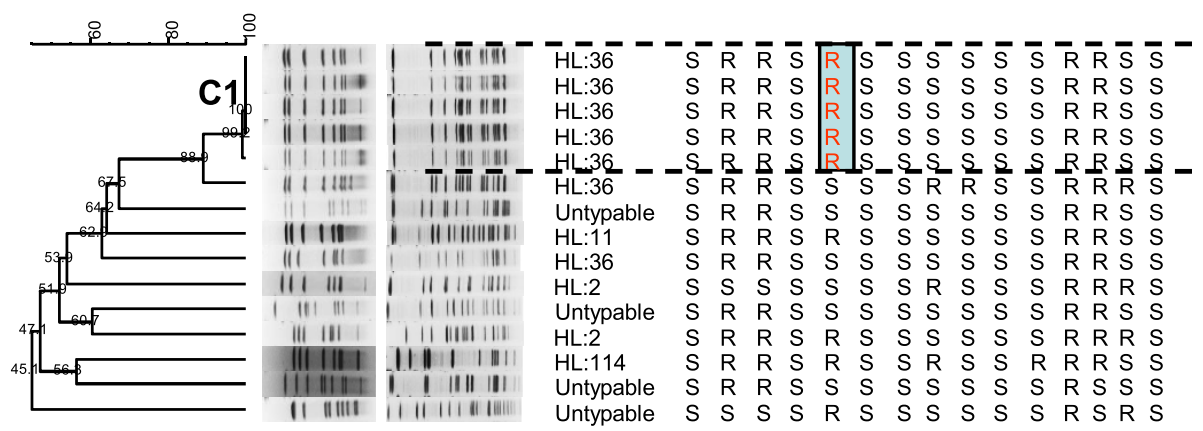

Figure 4 Dendrogram of 15 C. jejuni isolates with Lior serotypes and antimicrobial resistance from Sakaew 2002. Dendrogram C: PFGE cluster analysis of a Smal/Kpnl restriction enzyme digest of genomic DNA of 15 C. jejuni isolates from the Cobra Gold exercises (Sakaew, 2002) with evidence of clonality due to similarity in the PFGE patterns (>99.2\%), identical Lior serotype (HL:36) and a specific antimicrobial susceptibility pattern (genotype $\mathrm{C} 1$ with ampicillin resistance). $\mathrm{R}$, resistance; S, susceptible.

over a 2-year period, a similar low correlation was observed between serotype and antimicrobial resistance. Our data suggest that these patients became infected with unrelated $C$. jejuni isolates. Comprehensive monitoring of human C. jejuni isolates, including animal and environmental sources, should be expanded in Thailand to monitor antimicrobial resistance and to better document potential sources of infection.

\section{Methods}

\section{Sources of isolates}

Under approved human use protocols, stool specimens were obtained from patients with diarrhea and from asymptomatic controls in an acute diarrhea study among foreign travelers from highly developed countries at Bumrungrad Hospital in Bangkok, Thailand during 2001-2002. Stool specimens were collected from U.S.

\section{Dendrogram D}

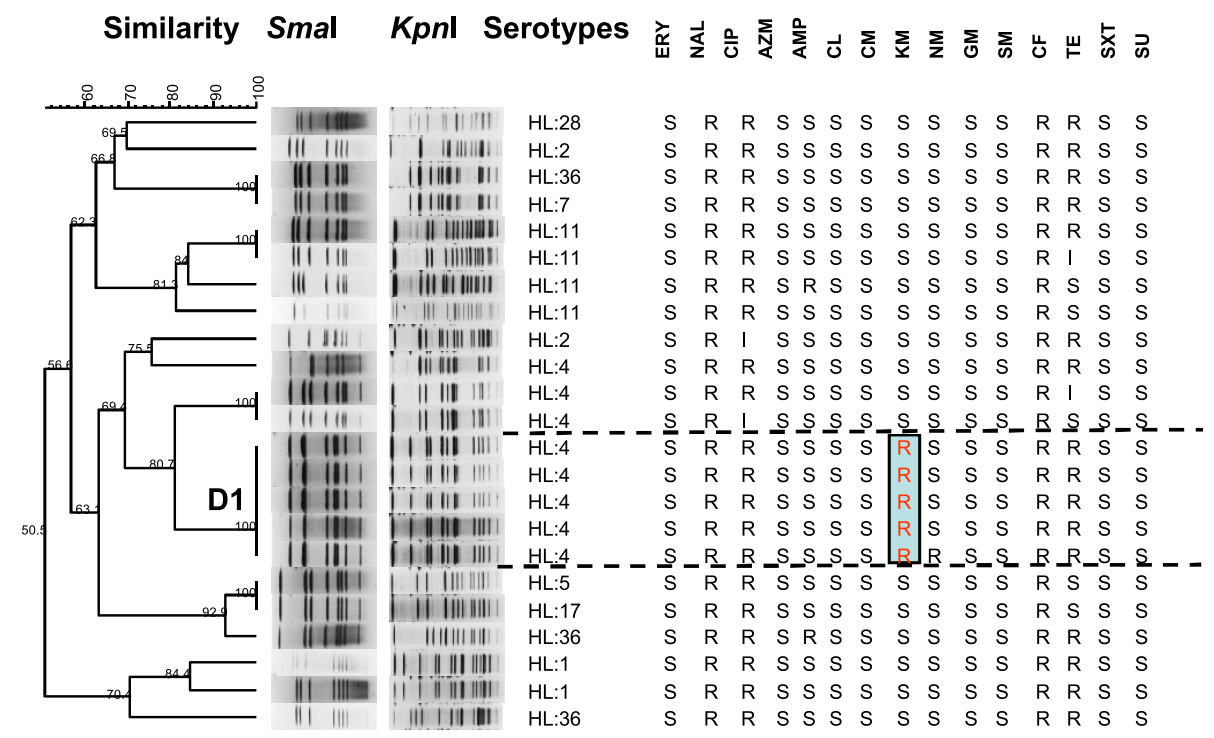

Figure 5 Dendrogram of 23 C. jejuni isolates with Lior serotypes and antimicrobial resistance from Pranburi 2003. Dendrogram D: PFGE cluster analysis of a Smal/Kpnl restriction enzyme digest of genomic DNA of 23 C. jejuni isolates from the Cobra Gold exercises (Pranburi, 2003) with evidence of clonality due to similarity in the PFGE patterns (100\%), identical Lior serotype (HL: 4) and specific antimicrobial susceptibility patterns (genotype D1 with additional kanamycin resistance). R, resistance; I, intermediate susceptibility; S, susceptible. 
soldiers with acute diarrhea and from asymptomatic controls; the soldiers were deployed for the Cobra Gold exercises lasting one for four weeks at different sites in Thailand during 1998-2003. Only C. jejuni isolates from acute diarrhea cases were included in this study. Table 1 describes the number of $C$. jejuni isolates from cases in each study location.

\section{Isolation and identification}

All stool specimens were cultured for Campylobacter spp. using a modified filtration method described previously [41]. Suspected colonies, growing on Brucella Agar (Difco, Detroit, MI, USA) with 5\% sheep blood (BAP), were identified as Campylobacter spp. by colony characteristics, Gram staining, oxidase tests, and catalase tests, followed by phenotypic tests including hippurate hydrolysis, nitrate reduction, $\mathrm{H}_{2} \mathrm{~S}$ TSI, oxygen tolerance, and microaerobic growth at $25^{\circ} \mathrm{C}, 37^{\circ} \mathrm{C}$, and $42^{\circ} \mathrm{C}$. C. jejuni isolates were differentiated from C. coli by the hippurate hydrolysis test. All $C$. jejuni isolates were kept in glycerol medium at $-70^{\circ} \mathrm{C}$ for further analysis.

\section{Serotyping}

Lior serotyping was performed by an agglutination assay with specific antiserum obtained from the National Laboratory for Enteric Pathogens (NLEP) in Winnipeg, Manitoba, Canada. These antisera were routinely used to serotype $C$. jejuni and $C$. coli isolates at AFRIMS. The antisera detect heat-labile antigens [9] and identify 33 common HL serotypes.

\section{Antimicrobial susceptibility testing}

C. jejuni isolates were tested for susceptibility to antimicrobial drugs using a disk diffusion assay as described previously [42], with modifications. BAP subcultures of patient isolates at 18- to 48-h were suspended in Mueller Hinton broth (BD Diagnostic Systems, Sparks, MD, USA.) to obtain a turbidity equivalent to a $1.0 \mathrm{McFar}-$ land standard, and suspensions were inoculated onto Mueller Hinton II agar supplemented with 5\% sheep blood. At the time of each study, all $C$. jejuni isolates were tested for susceptibility to the following antimicrobials (BD Diagnostic Systems): NAL $(30 \mu \mathrm{g})$, CIP $(5 \mu \mathrm{g})$, ERY $(15 \mu \mathrm{g})$, and AZM $(15 \mu \mathrm{g})$. The $138 \mathrm{C}$. jejuni isolates in 2001-2003 were further tested for susceptibility to 11 additional antimicrobials by disk diffusion assay. These antimicrobial disks included AMP $(10 \mu \mathrm{g}), \mathrm{CM}$ $(30 \mu \mathrm{g})$, KM $(30 \mu \mathrm{g}), \mathrm{GM}(10 \mu \mathrm{g}), \mathrm{SM}(10 \mu \mathrm{g})$, TE $(30$ $\mu \mathrm{g}), \mathrm{SXT}(1.25 / 23.75 \mu \mathrm{g}), \mathrm{SU}(250 \mu \mathrm{g}), \mathrm{CL}(10 \mu \mathrm{g}), \mathrm{NM}$ $(30 \mu \mathrm{g})$, and CF $(30 \mu \mathrm{g})$. Disks were placed on the surfaces of inoculated Mueller Hinton II agar plates. Inoculated plates were incubated at $37^{\circ} \mathrm{C}$ for $24 \mathrm{~h}$ in a microaerobic environment. The plates were re-incubated up to $48 \mathrm{~h}$ if insufficient growth of $C$. jejuni isolates on the Muller Hinton II agar plates was obtained at $24 \mathrm{~h}$. Because no standardized interpretive criteria exist for Campylobacter spp., the inhibition zone diameters were measured and interpreted following the disk manufacturer's instructions and compared against the Clinical and Laboratory Standards Institute (formerly NCCLS) standard guidelines for aerobic gram-negative bacilli to interpret the results as susceptible, intermediate, or resistant [43]. Escherichia coli ATCC 25922 and Staphylococcus aureus ATCC 25923 were used as standard organisms for all disk diffusion assays.

\section{Statistical analysis}

Pearson's chi-square tests of independence for the antimicrobial susceptibility data (NAL, CIP, ERY, AZM, TE, SXT, AMP, and KM) between locations were performed using the Monte Carlo-exact (2-sided) method in SPSS version 12.0 (SPSS Inc., Chicago, IL, USA). A p-value $<0.05$ was considered significant.

\section{PFGE}

PFGE was performed according to the One-Day (24-28 h) Standardized Laboratory Protocol for Molecular Subtyping by the CDC [12] with the minor modifications described below. The cell density of each isolate was adjusted to an O.D. of 0.6 using a spectrophotometer (Spectramax 190; Molecular Devices, Sunnyvale, CA, USA) that was different from the spectrophotometers suggested by the CDC. The PFGE patterns were analyzed to generate dendrograms of the combined SmaI and $K p n \mathrm{I}$ similarities using BioNumerics version 5.0 (Applied Maths, Sint-Martens-Latem, Belgium) by UPGMA type and Dice coefficient with $1.5 \%$ optimization and tolerance. Dendrograms were made for the composite data of all of the C. jejuni isolates and for the different locations.

\section{Abbreviations}

The following abbreviations were used: RFLP: restriction fragment length polymorphism; RAPD: random amplification of polymorphic DNA; PFGE: pulsed-field gel electrophoresis; AFLP: amplified fragment length polymorphism; MLST: multilocus sequence typing; MIC: minimal inhibitory concentration; HL: heat-labile; NAL: nalidixic acid; CIP: ciprofloxacin; ERY erythromycin; AZM: azithromycin; AMP: ampicillin; CM: chloramphenicol; KM: kanamycin; GM: gentamicin; SM: streptomycin; TE: tetracycline; SXT:

trimethoprim-sulfamethoxazole; SU: sulfisoxazole; CL: colistin; NM: neomycin; CF: cephalothin.

\section{Acknowledgements}

All of the study projects described herein were supported financially by the Military Infectious Diseases Research Program, United States Army Medical Research and Materiel Command in Fort Detrick, MD, USA. We are grateful to the National Laboratory for Enteric Pathogens in Winnipeg, Manitoba, Canada for providing antiserum to perform the Lior serotyping. The views expressed here are those of the authors and are not to be construed as reflecting the views of the United States Department of Defense or the United States Army. 


\section{Author details}

'Department of Enteric Diseases, Armed Forces Research Institute of Medical Sciences, 315/6 Rajvithi Road, Phayathai, Bangkok 10400, Thailand. ${ }^{2}$ Department of Veterinary Pathobiology, Faculty of Life Sciences, Copenhagen University, Stigboejlen 4, DK-1870 Frederiksberg C, Denmark. ${ }^{3}$ Naval Medical Research Center, 503 Robert Grant Ave, Silver Spring, MD 20910, USA. ${ }^{4}$ Uniformed Services University of the Health Sciences, 4301 Jones Bridge Road, Bethesda, MD 20814-5119, USA. ${ }^{5}$ Bumrungrad Hospital, 33 Sukhumvit Soi 3, Bangkok 10110, Thailand.

\section{Authors' contributions}

OS designed and carried out the study project (including the data analysis and preparation of the draft manuscript), PP performed the PFGE of the C. jejuni isolates, $\mathrm{AD}$ provided ideas and comments on the draft manuscript, LB analyzed the epidemiological data for the $C$. jejuni isolates, PG provided expertise on the molecular biology of C. jejuni, DT directed the patient recruitment in the US military exercise, SA supported the study project etiology of acute diarrhea at Bumrungrad Hospital, and CJM conceived the idea for the study (and performed statistical analysis and worked on the final manuscript). All authors read and approved the final manuscript.

\section{Competing interests}

The authors declare that they have no competing interests.

Received: 4 October 2010 Accepted: 10 November 2010 Published: 10 November 2010

\section{References}

1. Bodhidatta L, Vithayasai N, Eimpokalarp B, Pitarangsi C, Serichantalergs O, Isenbarger DW: Bacterial enteric pathogens in children with acute dysentery in Thailand: increasing importance of quinolone-resistant Campylobacter. Southeast Asian J Trop Med Public Health 2002, 33:752-757.

2. Friedman CR, Neimann J, Wegener HC, Tauxe RV: Epidemiology of Campylobacter jejuni infections in the United States and other industrialized nations. In Campylobacter. 2 edition. Edited by: Nachamkin I, Blaser MJ. Washington, DC, USA: ASM Press; 2000:121-138.

3. Hoge CW, Gambel JM, Srijan A, Pitarangsi C, Echeverria P: Trends in antibiotic resistance among diarrheal pathogens isolated in Thailand over 15 years. Clin Infect Dis 1998, 26:341-345.

4. Taylor DN, Echeverria P, Pitarangsi C, Seriwatana J, Bodhidatta L, Blaser MJ: Influence of strain characteristics and immunity on the epidemiology of Campylobacter infections in Thailand. J Clin Microbiol 1988, 26:863-868.

5. Engberg J, Aarestrup FM, Taylor DE, Gerner-Smidt P, Nachamkin I: Quinolone and macrolide resistance in Campylobacter jejuni and C. coli: resistance mechanisms and trends in human isolates. Emerg Infect Dis 2001, 7:24-34.

6. Kang YS, Cho YS, Yoon SK, Yu MA, Kim CM, Lee JO, Pyun YR: Prevalence and antimicrobial resistance of Campylobacter jejuni and Campylobacter coli isolated from raw chicken meat and human stools in Korea. J Food Prot 2006, 69:2915-2923.

7. Harvey SM: Hippurate hydrolysis by Campylobacter fetus. J Clin Microbiol 1980, 11:435-437.

8. Frost JA, Oza AN, Thwaites RT, Rowe B: Serotyping scheme for Campylobacter jejuni and Campylobacter coli based on direct agglutination of heat-stable antigens. J Clin Microbiol 1998, 36:335-339.

9. Lior H, Woodward DL, Edgar JA, Laroche LJ, Gill P: Serotyping of Campylobacter jejuni by slide agglutination based on heat-labile antigenic factors. J Clin Microbiol 1982, 15:761-768.

10. Penner $\mathrm{JL}$, Hennessy JN: Passive hemagglutination technique for serotyping Campylobacter fetus subsp. jejuni on the basis of soluble heat-stable antigens. J Clin Microbiol 1980, 12:732-737.

11. Newell DG, Frost JA, Duim B, Wagenaar JA, Madden RH, van der Plas J, On SLW: New developments in the subtyping of Campylobacter species. In Campylobacter. 2 edition. Edited by: Nachamkin I, Blaser MJ. Washington, DC, USA: ASM Press; 2000:27-44.

12. Centers for Disease Control and Prevention: One-day (24-28 h) standardized laboratory protocol for molecular subtyping of Escherichia coli 0157:H7, non-typhoidal Salmonella serotypes, and Shigella sonnei by pulsed-field gel electrophoresis (PFGE). PulseNet 8th Annual Update Meeting; April 27-30; San Diego, CA Centers for Disease Control and Prevention; 2004.
13. Ribot EM, Fitzgerald C, Kubota K, Swaminathan B, Barrett TJ: Rapid pulsedfield gel electrophoresis protocol for subtyping of Campylobacter jejuni. J Clin Microbiol 2001, 39:1889-1894.

14. Fitzgerald C, Stanley K, Andrew S, Jones K: Use of pulsed-field gel electrophoresis and flagellin gene typing in identifying clonal groups of Campylobacter jejuni and Campylobacter coli in farm and clinical environments. Appl Environ Microbiol 2001, 67:1429-1436.

15. Nielsen EM, Engberg J, Fussing V, Petersen L, Brogren CH, On SL: Evaluation of phenotypic and genotypic methods for subtyping Campylobacter jejuni isolates from humans, poultry, and cattle. I Clin Microbiol 2000, 38:3800-3810.

16. Zorman T, Heyndrickx M, Uzunovic-Kamberovic S, Smole Mozina S: Genotyping of Campylobacter coli and C. jejuni from retail chicken meat and humans with campylobacteriosis in Slovenia and Bosnia and Herzegovina. Int J Food Microbiol 2006, 110:24-33.

17. Hanninen ML, Pajarre S, Klossner ML, Rautelin H: Typing of human Campylobacter jejuni isolates in Finland by pulsed-field gel electrophoresis. J Clin Microbiol 1998, 36:1787-1789.

18. Peacock S, de Silva G, Justice A, Cowland A, Moore C, Winearls C, Day N: Comparison of multilocus sequence typing and pulsed-field gel electrophoresis as tools for typing Staphylococcus aureus isolates in a microepidemiological setting. I Clin Microbiol 2002, 40:3764-3770.

19. Sails AD, Swaminathan B, Fields PI: Utility of multilocus sequence typing as an epidemiological tool for investigation of outbreaks of gastroenteritis caused by Campylobacter jejuni. J Clin Microbiol 2003, 41:4733-4739.

20. Beecham HJ, Lebron Cl, Echeverria P: Short report: impact of traveler's diarrhea on United States troops deployed to Thailand. Am J Trop Med Hyg 1997, 57:699-701.

21. Isenbarger DW, Hoge CW, Srijan A, Pitarangsi C, Vithayasai N, Bodhidatta L, Hickey KW, Cam PD: Comparative antibiotic resistance of diarrheal pathogens from Vietnam and Thailand, 1996-1999. Emerg Infect Dis 2002, 8:175-180.

22. Sanders JW, Isenbarger DW, Walz SE, Pang LW, Scott DA, Tamminga C, Oyofo BA, Hewitson WC, Sanchez JL, Pitarangsi C, Echeverria P, Tribble DR: An observational clinic-based study of diarrheal illness in deployed United States military personnel in Thailand: presentation and outcome of Campylobacter infection. Am J Trop Med Hyg 2002, 67:533-538.

23. Tribble DR, Baqar S, Pang LW, Mason C, Houng HS, Pitarangsi C, Lebron C, Armstrong A, Sethabutr O, Sanders JW: Diagnostic approach to acute diarrheal illness in a military population on training exercises in Thailand, a region of Campylobacter hyperendemicity. J Clin Microbiol 2008, 46:1418-1425.

24. Serichantalergs O, Dalsgaard A, Bodhidatta L, Krasaesub S, Pitarangsi C, Srijan A, Mason CJ: Emerging fluoroquinolone and macrolide resistance of Campylobacter jejuni and Campylobacter coli isolates and their serotypes in Thai children from 1991 to 2000. Epidemiol Infect 2007, 135:1299-1306.

25. Woodward DL, Rodgers FG: Identification of Campylobacter heat-stable and heat-labile antigens by combining the Penner and Lior serotyping schemes. J Clin Microbiol 2002, 40:741-745.

26. Roumagnac P, Gagnevin L, Pruvost O, Achtman M: Insights into structure and evolution of bacterial species that are revealed by molecular methods. In Encyclopedia of Infectious Diseases: Modern Methodologies. Edited by: Tibayrenc M. Hoboken, NJ, USA: John Wiley 2007:475-494.

27. Li CC, Chiu CH, Wu JL, Huang YC, Lin TY: Antimicrobial susceptibilities of Campylobacter jejuni and coli by using E-test in Taiwan. Scand I Infect Dis 1998, 30:39-42.

28. Han K, Jang SS, Choo E, Heu S, Ryu S: Prevalence, genetic diversity, and antibiotic resistance patterns of Campylobacter jejuni from retail raw chickens in Korea. Int J Food Microbiol 2007, 114:50-59.

29. Gibreel A, Tracz DM, Nonaka L, Ngo TM, Connell SR, Taylor DE: Incidence of antibiotic resistance in Campylobacter jejuni isolated in Alberta, Canada, from 1999 to 2002, with special reference to tet(O)-mediated tetracycline resistance. Antimicrob Agents Chemother 2004, 48:3442-3450.

30. Taylor DE, Tracz DM: Mechanisms of antimicrobial resistance in Campylobacter. In Campylobacter Molecular and Cell Biology. Edited by: Ketley JM, Konkel ME. Norfolk, UK: Horizon Bioscience; 2005:193-204.

31. Boonmar S, Morita $Y$, Fujita M, Sangsuk L, Suthivarakom K, Padungtod $P$, Maruyama S, Kabeya H, Kato M, Kozawa K, Yamamoto S, Kimura H: Serotypes, antimicrobial susceptibility, and gyr $A$ gene mutation of 
Campylobacter jejuni isolates from humans and chickens in Thailand. Microbiol Immunol 2007, 51:531-537.

32. Gaudreau C, Gilbert H: Comparison of disc diffusion and agar dilution methods for antibiotic susceptibility testing of Campylobacter jejuni subsp. jejuni and Campylobacter coli. J Antimicrob Chemother 1997, 39:707-712.

33. Huysmans MB, Turnidge JD: Disc susceptibility testing for thermomphilic campylobacters. Pathology 1997, 29:209-216.

34. Luber P, Bartelt E, Genschow E, Wagner J, Hahn H: Comparison of broth microdilution, E Test, and agar dilution methods for antibiotic susceptibility testing of Campylobacter jejuni and Campylobacter coli. J Clin Microbiol 2003, 41:1062-1068.

35. McDermott PF, Bodeis-Jones SM, Fritsche TR, Jones RN, Walker RD: Broth microdilution susceptibility testing of Campylobacter jejuni and the determination of quality control ranges for fourteen antimicrobial agents. J Clin Microbiol 2005, 43:6136-6138.

36. Luangtongkum T, Morishita TY, El-Tayeb AB, Ison AJ, Zhang Q: Comparison of antimicrobial susceptibility testing of Campylobacter spp. by the agar dilution and the agar disk diffusion methods. J Clin Microbiol 2007, 45:590-594.

37. van der Beek MT, Claas EC, Mevius DJ, van Pelt W, Wagenaar JA, Kuijper EJ: Inaccuracy of routine susceptibility tests for detection of erythromycin resistance of Campylobacter jejuni and Campylobacter coli. Clin Microbiol Infect 2010, 16:51-56.

38. Serichantalergs $\mathrm{O}$, Jensen $\mathrm{LB}$, Pitarangsi C, Mason CJ, Dalsgaard A: A possible mechanism of macrolide resistance among multiple resistant Campylobacter jejuni and Campylobacter coli isolated from Thai children during 1991-2000. Southeast Asian J Trop Med Public Health 2007, 38:501-506.

39. Cardinale E, Rose V, Perrier Gros-Claude JD, Tall F, Rivoal K, Mead G, Salvat G: Genetic characterization and antibiotic resistance of Campylobacter spp. isolated from poultry and humans in Senegal. J Appl Microbiol 2006, 100:209-217.

40. Ronner AC, Borch E, Kaijser B: Genetic profiling of Campylobacter jejuni strains from humans infected in Sweden or in Thailand, and from healthy Swedish chickens, studied by pulsed-field gel electrophoresis (PFGE). Scand J Infect Dis 2005, 37:579-584.

41. Steele TW, McDermott SN: The use of membrane filters applied directly to the surface of agar plates for the isolation of Campylobacter jejuni from feces. Pathology 1984, 16:263-265.

42. Bauer AW, Kirby WM, Sherris JC, Turck M: Antibiotic susceptibility testing by a standardized single disk method. Tech Bull Regist Med Technol 1966, 36:49-52.

43. National Committee for Clinical Laboratory Standards: Performance standards for antimicrobial susceptibility testing. Eighth informational supplement. NCCLS document M100-S08 Wayne, PA, USA: National Committee for Clinical Laboratory Standards; 1998.

doi:10.1186/1757-4749-2-15

Cite this article as: Serichantalergs et al:: PFGE, Lior serotype, and antimicrobial resistance patterns among Campylobacter jejuni isolated from travelers and US military personnel with acute diarrhea in Thailand, 1998-2003. Gut Pathogens 2010 2:15.

\section{Submit your next manuscript to BioMed Central and take full advantage of:}

- Convenient online submission

- Thorough peer review

- No space constraints or color figure charges

- Immediate publication on acceptance

- Inclusion in PubMed, CAS, Scopus and Google Scholar

- Research which is freely available for redistribution

Submit your manuscript at www.biomedcentral.com/submit 